\title{
September 2015 Critical Care Case of the Month: If You Don't Look, You Won't Find
}

\author{
Robert A. Raschke, MD
}

\author{
Banner University Medical Center \\ Phoenix, AZ
}

\begin{abstract}
History of Present IIIness
A 55-year-old woman was transferred from Mexico emergently for acute cardiomyopathy. On the day of admission, she went for a 45-min "exercise" walk and cleaned her house. Afterwards, while taking a shower, she suffered an acute onset of dyspnea with nausea and vomiting and possibly a small amount of hematemesis. She appeared seriously ill to her husband, who took her blood pressure (198/?) and pulse (90) and rushed her to a local medical facility. There, she was found to have severe pulmonary edema, and a troponin of 11 . Her echo showed inferior wall motion abnormality with an ejection fraction of $35 \%$. However, coronary catheterization showed normal coronaries. She was treated with oxygen, furosemide, labetolol and enoxaparin and transferred emergently to Banner-University Medical Center.
\end{abstract}

\section{Past Medical History, Family History and Social History}

The patient reported intermittent "spells" since May. These typically occurred upon lying down in bed and were characterized by her as a feeling of "numbness" or tingling which ascends from her chest to her head associated with palpitations and a feeling of "desperation", typically relieved after a few minutes upon getting up out of bed. She had a history of hypertension and had been on losartan but this was discontinued a few months previously because of the onset of orthostatic dizziness. She also has a history of hypothyroidism and is taking synthroid. She was treated three times in the last 6 month for amoebiasis. She is a medical missionary to La Paz, Mexico and has recently traveled to Bolivia and Guatemala.

\section{Review of Systems}

She has had some night sweats, coughing with deep inspiration, and some slight hemoptysis. She did have a headache one month previously at $7000 \mathrm{ft}$ elevation while in Guatemala.

\section{Physical Examination}

- She appears in moderate distress. Her vital signs are normal other than a mild tachycardia.

- She does have rales on auscultation of her lungs.

- The remainder of the physical examination was unremarkable.

\section{Radiography}

A portable chest radiograph is performed (Figure 1). 


\section{UPRIGHT}

Figure 1. Admission portable chest radiograph.

\section{Laboratory evaluation}

Her CBC shows a normal hemoglobin and hematocrit but with an elevated white blood cell count of 26,500 cells $/ \mathrm{mcL}$ with a left shift. Admission electrolytes and blood sugar are within normal limits.

What additional procedures/testing are indicated?

1. Blood cultures

2. Echocardiogram

3. Electrocardiogram

4. NT-pro-brain natriuretic peptide (NT-pro-BNP)

5. All of the above 


\section{Correct!}

\section{All of the above}

All were performed. Her blood cultures had no growth. Plasma NT-pro-BNP was markedly elevated at $17,685 \mathrm{pg} / \mathrm{ml}$ (normal $<300$ under the age of 50 ). Her echocardiogram showed inferior septum regional hypokinesis with an ejection fraction of 45\%. Her electrocardiogram is shown in Figure 2.

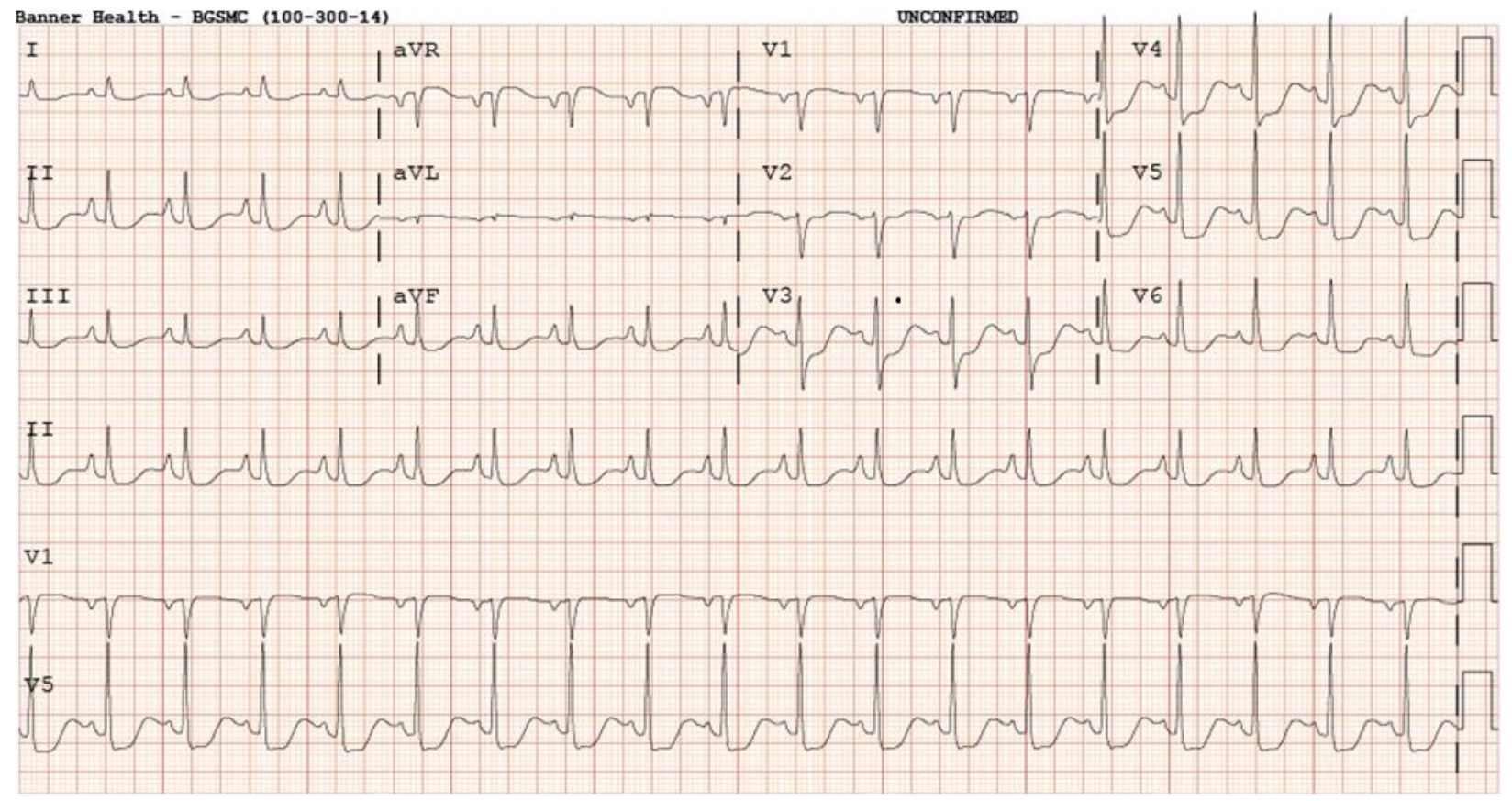

Figure 2. Admission electrocardiogram.

In addition, her troponin was elevated at $18.9 \mathrm{ng} / \mathrm{mL}$ (normal $<0.1$ ), C-reactive protein $299 \mathrm{mg} / \mathrm{dL}$ (normal <10) and creatine phosphokinase $760 \mathrm{U} / \mathrm{L}$ (normal <198)..

\section{Which additional tests are indicated?}

1. ELISA for IgM antibodies against Coxsackie virus

2. ELISA for $T$. cruzi

3. Peripheral blood smear

4. Viral stool culture

5. All of the above 


\section{Correct! \\ 5. All of the above}

The patient has subacute myocarditis. Chagas disease, caused by the parasite Trypanosoma cruzi is endemic to Latin and South America and is a common cause of myocarditis. However, Chagas disease is characterized by a chronic dilated cardiomyopathy. An acute presentation as seen in our patient, is distinctly rare with $\mathrm{T}$ cruzi. which can be diagnosed by a blood smear but usually requires antibody confirmation. In the US, viral myocarditis is more common and coxsackie, adeno and parvo viruses being common causes. However, her work up for any infectious cause was negative.

She rapidly improved clinically with further treatment with furosemide and carvedilol. Losartan was started but discontinued because her blood pressure was typically in the $90 / 60 \mathrm{mmHg}$ range. An MRI of her heart was consistent with myocarditis, with an EF of $55 \%$. Endomyocardial biopsy was considered unnecessary due to her rapid improvement. Discharge was planned with an ambulatory event monitor, since no episode of arrhythmia had been observed to explain the periodic "spells" that occurred before her sudden episode of deterioration.

However, just prior to discharge, a clinician recalled the patient's husband's report of a blood pressure of 198/? $\mathrm{mmHg}$ during her episode of deterioration and questioned the diagnosis of pheochromocytoma.

Based on the clinical presentation, which measurements are indicated next?

1. Free plasma metanephrines or urinary metanephrines

2. 5-hydroxyindoleacetic acid (5-HIAA) in the urine

3. Abdominal computerized tomography

4. 1 and 3

5. All of the above 


\section{Correct! \\ 1. Free plasma metanephrines or urinary metanephrines}

Controversy persists regarding the best screening test for pheochromocytoma, Studies have demonstrated that plasma fractionated metanephrines have the highest sensitivity, and thereby the best NPV, and 24-hour urine for fractionated metanephrines has the best specificity and therefore the highest PPV. Our patient's free plasma metanephrines were $402 \mathrm{pg} / \mathrm{mL}$ (normal <57) and free plasma normetanephrines $1327 \mathrm{pg} / \mathrm{mL}$ (normal $<148$ ). Since only about 1 in 300 patients evaluated for pheochromocytoma will turn out to have it, CT scanning would not be a cost effective screening tool. Urine 5hydroxyindoleacetic acid (5-HIAA) is a diagnostic test for carcinoid tumor. Clinically carcinoid tumors present more commonly with "dry" flushing (flushing with no sweating) and nocturnal diarrhea.

The most important thing about pheochromocytoma is to clinically recognize when to suspect and test for it. . As many as $50-75 \%$ of pheochromocytomas demonstrable on autopsy were never clinically diagnosed. The classic triad of episodic headaches, diaphoresis and tachycardia is seen in only $25 \%$ of patients with pheochromocytoma (1). About $80-90 \%$ of patients have hypertension but $40-50 \%$ have only episodic hypertension. Orthostasis and acute cardiomyopathy such as seen in our patient are less common, but well described. Patient's presenting with acute cardiomyopathy secondary to pheochromocytoma famously deteriorate if given beta-blockers, due to unopposed alpha sympathomimetic effect Another uncommon but classic presentation for a patient with undiagnosed pheochromocytoma is unexpected life-threatening hyper or hypotension during an unrelated surgical procedure.

Our patient underwent CT of the abdomen which showed a left adrenal mass consistent with pheochromocytoma (Figure 3).

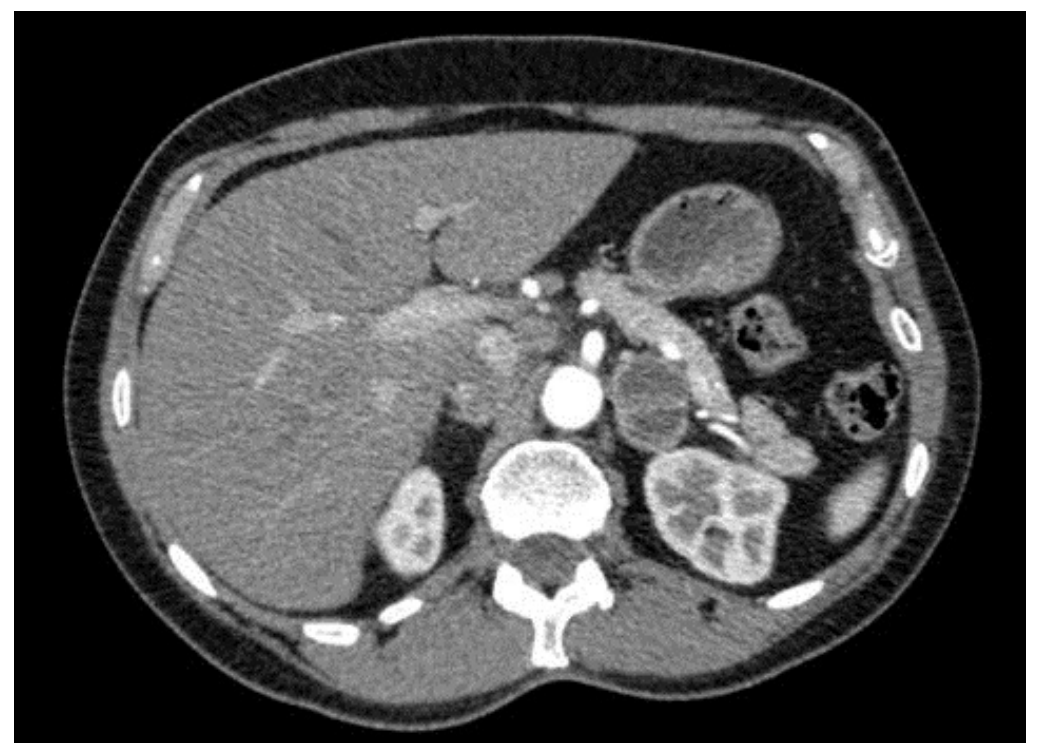

Figure 3. Representative view of contrast enhanced abdominal CT scan. 
About $10 \%$ of pheochromocytomas are multiple, about $10 \%$ are extra-adrenal and about $10 \%$ are malignant - but radiographical evaluation did not reveal evidence of any of these. The patient was treated with phenoxybenzamine (a non-competitive ganglionic alpha blocker) and then labetolol - only after alpha blockade was established. The patient was also put on a high sodium diet to help ameliorate volume contraction associated with untreated pheochromocytoma. It typically takes at least two weeks to prepare a pheochromocytoma patient for adrenalectomy .

The take home points of this presentation are:

- Pheochromocytoma can present as a pseudo-myocardial infarction / pseudomyocarditis type of syndrome

- History-taking is never complete

- You will never diagnose a pheo if you do not consider it and test for it.

\section{Reference}

1. Zuber SM, Kantorovich V, Pacak K. Hypertension in pheochromocytoma: characteristics and treatment. Endocrinol Metab Clin North Am. 2011;40(2):295311. [CrossRef] [PubMed] 